\title{
Encurtamento pelo frio de fibras musculares oxidativas de bovinos pela técnica de NADH-TR
}

\author{
Cold shortening on bovine oxidative muscle fibers by NADH-TR
}

\author{
Juliana Maria Pereira Felício Gonfiantini Fernandes ${ }^{1}$ Marcos Franke Pinto $^{2}$ \\ Elisa Helena Giglio Ponsano ${ }^{2}$ Ana Paula da Silva Almeida ${ }^{3}$ Urbano Gomes Pinto de Abreu $^{4}$ \\ Jorge Antonio Ferreira de Lara ${ }^{4^{*}}$
}

\section{RESUMO}

\begin{abstract}
Carcaças bovinas resfriadas rapidamente podem apresentar uma contração muscular conhecida como encurtamento pelo frio. Esse fenômeno, prejudicial à textura da carne, ocorre principalmente nas fibras musculares oxidativas. $O$ objetivo deste trabalho foi disponibilizar uma ferramenta analítica para distinguir essas fibras e determinar com maior precisão a contração do tecido muscular pela mensuração do comprimento dos sarcômeros. Amostras do músculo Longissimus dorsi de 12 novilhas nelore foram coletadas. As amostras obtidas de uma das meias-carcaças foram submetidas a uma refrigeração rápida, e as amostras provenientes da outra meia-carcaça à refrigeração lenta. Foi testado um método analítico, baseado na técnica de coloração por Nicotinamida Adenina Dinucleotídeo - Tetrazolium Redutase (NADH-TR), para mensurar, por microscopia, o comprimento dos sarcômeros das fibras vermelhas. Foram determinadas as velocidades de queda de $\mathrm{pH}$ e temperatura, a área do olho de lombo (AOL) e a força de cisalhamento. Os resultados demonstraram que a temperatura é o principal fator responsável pelo comprimento do sarcômero quando a velocidade de resfriamento é rápida, sendo essa influência menor quando a queda de temperatura é mais lenta. Desta forma, demonstrou-se que a técnica de coloração com NADHTR é capaz de detectar a ocorrência do encurtamento pelo frio nos músculos esqueléticos de bovinos.
\end{abstract}

Palavras-chave: fibras musculares, refrigeração de carnes, qualidade da carne.

\section{ABSTRACT}

Bovine carcasses quickly cooled may develop a muscle contraction known as cold shortening. This process,

\begin{abstract}
harmful to meat texture, occurs mainly in the oxidative muscle fibers. This work was aimed at studing an analytical tool to distinguish these fibers and to evaluate, more precisely, the muscle contraction through the measurement of sarcomeres length. Longissimus dorsi muscles of 12 heifers were used as samples. Samples from one of the half carcasses were fast cooled and the samples from the others half carcasses were slowly cooled. An analytical technique based on Nicotinamide Adenine Dinucleotide - Tetrazolium Redutase (NADH-TR) methodology was performed to measure, by microscopy, the red fibers sarcomeres lenghts. Temperature and $\mathrm{pH}$ drops in the samples, shear force and rib eye area were also evaluated. Results pointed out that the temperature is the main responsible for the sarcomere length in the fast cooling and that, when slow cooling is performed, the influence of this parameter is less important. These results indicate that the NADH-TR technique is able to estimate cold shortening occurrence in bovine skeletal muscles.
\end{abstract}

Key words: meat quality, muscle fibers.

\section{INTRODUÇÃO}

A maciez é reconhecidamente um atributo importante para a qualidade da carne bovina. Por ser essa uma característica influenciada por muitos fatores, é de difícil previsão antes do momento do consumo (KOOHMARAIE, 1994). Um fator freqüentemente associado à maciez é o grau de contração do tecido muscular, causado pela exposição do músculo a baixas temperaturas antes do estabelecimento do rigor mortis,

${ }^{1}$ Curso de Pós-graduação em Ciência Animal, departamento de Zootecnia. Universidade Federal de Mato Grosso do Sul (UFMS), Campo Grande, MS, Brasil.

${ }^{2}$ Departamento de Produção e Saúde Animal, Universidade Estadual Paulista (UNESP), Araçatuba, SP, Brasil.

${ }^{3}$ Curso de Pós-graduação em Ciência Animal, curso de Medicina Veterinária, UNESP, Araçatuba, SP, Brasil.

${ }^{4}$ Empresa Brasileira de Pesquisa Agropecuária, EMBRAPA Pantanal. Rua 21 de Setembro, 1880, CP 109, 79320-900, Corumbá,

MS, Brasil. E-mail: jorge @ cpap.embrapa.br. Autor para correspondência. 
fenômeno conhecido como cold shortening ou encurtamento pelo frio (PARRISH et al., 1973). O tecido muscular sofre maior encurtamento quando está em pré-rigor e na temperatura entre 2 e $37^{\circ} \mathrm{C}$, exceto na faixa de temperatura entre 14 e $19^{\circ} \mathrm{C}$, onde o encurtamentoé mínimo (LOCKER \& HAGYARD, 1963).

HERTZMAN et al. (1993) concordam que, quando a temperatura no início do rigor situa-se entre 15 e $20^{\circ} \mathrm{C}$, o encurtamento é mínimo (aproximadamente $10 \%$ do comprimento muscular). Entre 20 e $40^{\circ} \mathrm{C}$, ocorre cerca de $30 \%$ e, entre 14 e $0{ }^{\circ} \mathrm{C}, 50 \%$ de encurtamento. Se a sobreposição dos miofilamentos resulta num encurtamento superior a $20 \%$, ocorre prejuízo perceptível da maciez. Esse efeito de endurecimento aumenta até um pico em torno de $40 \%$ de encurtamento. A eficiência das câmaras de resfriamento é uma preocupação das indústrias do setor, pela necessidade de acelerar o processamento, de reduzir perdas e de inibir o crescimento microbiano.

Com isso, o encurtamento pelo frio tem papel de destaque na textura da carne. Sabe-se que o fenômeno ocorre com maior intensidade nas fibras com atividade predominantemente oxidativa, ou fibras vermelhas (OLSSON et al., 1994). Caso a aferição do comprimento dos sarcômeros seja realizada sem diferenciação dos tipos de fibras que compõem o tecido, certamente haverá uma variabilidade dos resultados que podem comprometer a eficiência dessa ferramenta analítica (HEINEMANN et al., 2002).

A caracterização dos aspectos morfológicos, metabólicos, funcionais e a identificação dos diferentes tipos de fibras podem ser realizadas utilizando-se várias colorações e reações histoquímicas. Dentre as de uso mais comum na investigação de músculos, pode-se citar a reação com NADH-TR (Nicotinamida Adenina Dinucleotídeo Tetrazolium Reductase), utilizada para demonstrar as características metabólicas dos tipos de fibras (SILVA, 1999).

Neste trabalho, o objetivo foi avaliar a eficiência da técnica de coloração com NADH-TR para estimar o comprimento do sarcômero somente nas fibras oxidativas, em tecido muscular de bovinos.

\section{MATERIAL E MÉTODOS}

Obtenção e preparo das amostras

Foram utilizadas 12 novilhas nelore, com 4 dentes da $2^{\text {a }}$ dentição e apresentando parâmetros de peso, conformação e acabamento de carcaça para serem consideradas tipo B, de acordo com o Sistema Brasileiro de Tipificação (Brasil, 1989). Os abates foram realizados no Frigorífico Friboi Ltda., em Andradina, SP, de acordo com as normas e exigências do Serviço de Inspeção Federal (BRASIL, 1997).

Imediatamente após o abate, amostras do músculo Longissimus dorsi retiradas de uma das meiascarcaças, à altura da $12^{\mathrm{a}}$ costela, foram submetidas ao resfriamento rápido, em túnel de congelamento $\left(-25^{\circ} \mathrm{C}\right)$, até que a temperatura no interior das amostras atingisse $0^{\circ} \mathrm{C}$, sendo, em seguida, transferidas para câmara de refrigeração $\left(-1^{\circ} \mathrm{C} \mathrm{e}+1^{\circ} \mathrm{C}\right)$. As amostras retiradas das outras meias-carcaças foram submetidas à refrigeração lenta, em câmara à temperatura entre 5 e $10^{\circ} \mathrm{C}$ nas primeiras 9 horas, depois transferidas para câmara com temperatura entre $-1^{\circ} \mathrm{C}$ e $1^{\circ} \mathrm{C}$. Foi determinada a velocidade de queda de temperatura e $\mathrm{pH}$ nas primeiras 24 horas pós-abate, com a utilização de um potenciômetro portátil de inserção, com compensador e visor de temperatura, marca Mettler Toledo, modelo 1120-X. Em seguida, as amostras foram embaladas a vácuo em sacos de polietileno e congeladas a $-18^{\circ} \mathrm{C}$, seguindo para o laboratório, onde foram realizadas as aferições de área de olho de lombo e de comprimento do sarcômero, análises químico-bromatológica e da força de cisalhamento.

Área de olho de lombo

Após o descongelamento das amostras, o contorno do corte foi traçado em papel vegetal para depois ser determinada a área de olho de lombo (AOL) em sistema analisador de imagens.

\section{Análise químico bromatológica}

Após remoção da gordura ostensiva das amostras, o material restante foi triturado em processador de alimentos e submetido à determinação do teor de umidade, gordura, proteína bruta e cinzas, de acordo com a metodologia descrita pela AOAC (1997). As análises foram realizadas em triplicata.

Determinação do comprimento do sarcômero

Para a determinação do comprimento dos sarcômeros, $4 \mathrm{~g}$ da porção central de cada amostra foram homogeneizados em solução de $\mathrm{KCl} 1,0 \mathrm{M}$, na razão de 1:5 (p/v) por 5 minutos, em aparelho tipo Stomacher. Em seguida, a $5 \mathrm{ml}$ da suspensão obtida, foram adicionadas 3 gotas de uma solução preparada com $8 \mathrm{mg}$ de NADH, 10mg de Nitro Blue Tetrazolium e 10ml de Tampão Tris $0,2 \mathrm{M}$ em pH 7,4. Após incubação por $20 \mathrm{~min}$. a $37^{\circ} \mathrm{C}$, uma alíquota da suspensão foi colocada em lâmina, coberta por lamínula, para análise em microscópio óptico sob aumento de 1000X, acoplado ao sistema analisador de imagens LEICAQ550IW. Foram realizadas pelo menos 5 medições em campos diferentes para cada amostra e os resultados expressam a média dessas observações. 
Determinação da força de cisalhamento

A determinação da força de cisalhamento foi feita em Texturômetro TAXT2, equipado com célula de cisalhamento tipo Warner Bratzler. As peças foram descongeladas em estufa BOD a $4^{\circ} \mathrm{C}$ e submetidas a cozimento em forno com temperatura constante de $170^{\circ} \mathrm{C}$ até atingirem $70^{\circ} \mathrm{C}$ internamente. Essas peças, resfriadas à temperatura ambiente, foram embaladas em filme plástico e permaneceram em estufa BOD por 12 horas $/ 4^{\circ} \mathrm{C}$. Para a análise, foram utilizados pelo menos 6 cilindros de $1,27 \mathrm{~cm}$ de diâmetro, retirados no sentido longitudinal das fibras. A velocidade empregada foi de $5,0 \mathrm{~mm} \mathrm{seg}^{-1}$ e os resultados dos picos de força foram expressos como força de cisalhamento, em kg (POSTE, 1993).

Análise estatística

O delineamento utilizado foi inteiramente casualizado, em que cada amostra de m. longissimus dorsi constituiu uma unidade experimental. O nível de significância foi de 5\% para comparação das médias. Foram determinados os coeficientes de correlação linear de Pearson e o comprimento de miômero foi relacionado com a queda de temperatura e $\mathrm{pH}$ utilizando equação de regressão linear. O software foi o SAS (STATISTICALANALYSIS SYSTEM, 1997).

\section{RESULTADOS E DISCUSSÃO}

As amostras submetidas ao resfriamento rápido atingiram a temperatura média de $0,6^{\circ} \mathrm{C}$, no interior do músculo, já na primeira aferição, com três horas post mortem, evidenciando queda acentuada de temperatura, quando comparadas às amostras submetidas ao resfriamento lento, que ainda apresentavam temperatura média de $14,2^{\circ} \mathrm{C}$ e só atingiram médias de temperatura próximas a $0^{\circ} \mathrm{C}$ cerca de 24 horas após o abate (Figura 1).

A temperatura em que o músculo se encontra no momento do estabelecimento do rigor mortis é um dos fatores importantes na determinação de sua maciez (HANNULA \& POULANNE, 2004). FELÍCIO (1993) preconiza que, para prevenir a ocorrência do encurtamento pelo frio, as carcaças devem permanecer numa temperatura acima de $10^{\circ} \mathrm{C}$ até a ocorrência do rigor, quando o pH está em torno de 6,0, ou aproximadamente 10 horas após a sangria.

Em ambos os tratamentos, o pH das amostras caiu abaixo de 6,0 em torno de 9 horas post mortem, quando a temperatura média das amostras submetidas à refrigeração rápida e lenta era, respectivamente, de $-0,86^{\circ} \mathrm{Ce} 6,0^{\circ} \mathrm{C}$ (Figura 1). Esses valores indicam que as amostras do tratamento refrigeração lenta podem ter sofrido algum grau de encurtamento, pois, neste momento, as amostras estavam em pré-rigor, com o $\mathrm{pH}$ ainda em queda, e apresentavam as temperaturas abaixo de $14^{\circ} \mathrm{C}$, condição indicativa de encurtamento pelo frio (LOCKER \& HAGYARD, 1963).

As médias dos valores observados na análise químico bromatológica e na aferição da AOL não diferiram $(\mathrm{P}>0,05)$ entre as amostras submetidas à refrigeração rápida e lenta (Tabela 1).

Além disso, correlações significativas não foram encontradas entre esses parâmetros e o comprimento de sarcômero, medidos pelo coeficiente de Pearson em ambos os tratamentos. Neste caso, esses resultados são úteis para evidenciar que não houve diferença entre as médias de cada parâmetro químicobromatológico entre os tratamentos, não influenciando assim a ocorrência de encurtamento pelo frio entre as amostras da câmara e do túnel.

A velocidade de resfriamento das amostras influenciou o grau de contração do tecido muscular, conforme pode ser observado na tabela 1 . No entanto, esse encurtamento, caracterizado pelo menor comprimento dos sarcômeros, não influenciou a textura das amostras. HWANG et al. (2004) relataram resultados semelhantes, não encontrando diferença nos valores de força de cisalhamento quando carcaças foram expostas a temperaturas diferentes, embora

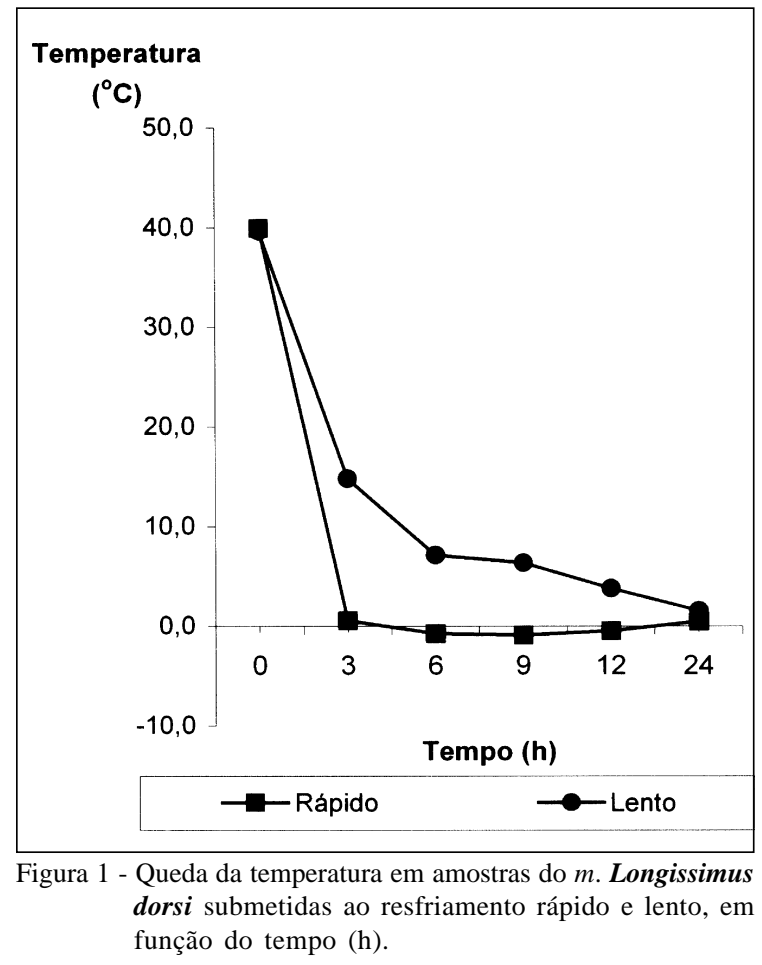

Ciência Rural, v.36, n.6, nov-dez, 2006. 
Tabela 1 - Comprimento de sarcômeros, força de cisalhamento (FC), análise químico bromatológica e área de olho de lombo (AOL) das amostras do m. Longissimus dorsi submetidas à refrigeração rápida e lenta.

\begin{tabular}{|c|c|c|c|c|c|c|c|}
\hline \multirow{2}{*}{$\begin{array}{l}\text { Velocidade } \\
\text { refrigeração }\end{array}$} & \multirow{2}{*}{ de } & Comprimento do sarcômero $(\mu \mathrm{m})$ & $\mathrm{FC}(\mathrm{kg})$ & Umidade $(\%)$ & Gordura (\%) & Proteínas $(\%)$ & $\operatorname{AOL}\left(\mathrm{cm}^{2}\right)$ \\
\hline & & $(\bar{x} \pm \mathrm{s})$ & $(\bar{x} \pm \mathrm{s})$ & $(\bar{x} \pm \mathrm{s})$ & $(\bar{x} \pm \mathrm{s})$ & $(\bar{x} \pm \mathrm{s})$ & $(\bar{x} \pm \mathrm{s})$ \\
\hline \multirow{2}{*}{ Rápida } & & $2,14 a$ & $12,14 \mathrm{a}$ & $71,31 \mathrm{a}$ & $7,44 a$ & $22,19 a$ & $50,55 \mathrm{a}$ \\
\hline & & $\pm 0,24^{1}$ & $\pm 1,44$ & $\pm 1,94$ & $\pm 4,60$ & $\pm 2,99$ & $\pm 9,64$ \\
\hline \multirow{2}{*}{ Lenta } & & $1,86 \mathrm{~b}$ & $13,23 \mathrm{a}$ & $71,42 \mathrm{a}$ & $5,04 \mathrm{a}$ & $21,64 \mathrm{a}$ & $52,27 \mathrm{a}$ \\
\hline & & $\pm 0,32^{2}$ & $\pm 1,96$ & $\pm 1,65$ & $\pm 2,33$ & $\pm 1,29$ & $\pm 9,92$ \\
\hline
\end{tabular}

Médias com letras minúsculas diferentes na coluna diferem $(\mathrm{P}>0,05)$ pelo teste de $\mathrm{F}$.

${ }^{1} \mathrm{CV}=11,2 \% \mathrm{e}^{2} \mathrm{CV}=17,2 \%$.

tenham observado diferença no comprimento dos sarcômeros. Os resultados relatados na literatura, utilizando o método tradicional de medida do comprimento de sarcômero da carne in natura, variam de 1,2 $\mu \mathrm{m}$ (WHEELER et al., 2002) a 1,7 $\mu \mathrm{m}$ (RHEE et al., 2004), diferenças que podem estar relacionadas ao método de resfriamento utilizado e à metodologia analítica empregada, sem diferenciação dos tipos de fibras musculares. HEINEMANN et al. (2002) enfatizam que o fenômeno do encurtamento pelo frio ocorre mais intensamente nas fibras oxidativas e que os métodos tradicionalmente empregados podem conduzir a um maior erro analítico.

$\mathrm{Na}$ figura 2, observa-se que o método de coloração possibilitou a diferenciação dos tipos de fibras do tecido muscular. Esse método de coloração utiliza as reações de óxido-redução do ciclo de Krebs. Por ação enzimática, o Nitro Blue Tetrazolium, sal de cor amarela, reduz-se, fica insolúvel e precipita, originando um composto de cor azul-violeta, a formazana. A enzima responsável por essa reação está presente nas regiões fibrilares internas, nas mitocôndrias e no retículo sarcoplasmático das células musculares. Portanto, as fibras oxidativas, por conterem essas estruturas e organelas em maior concentração, coram mais intensamente (SILVA, 1999).

Associando-se o comprimento do sarcômero (y) com pH e temperatura, foi possível obter as equações de regressão linear 1 e 2 para os tratamentos de refrigeração rápida e lenta, respectivamente.

EQUAÇÃO 1 (refrigeração rápida): $\mathrm{y}_{\mathrm{t}}=6,235-0,572 \mathrm{pH}$ $-1,158 \mathrm{~T}$, onde:

$\mathrm{y}_{\mathrm{t}}=$ comprimento de sarcômero para amostras do

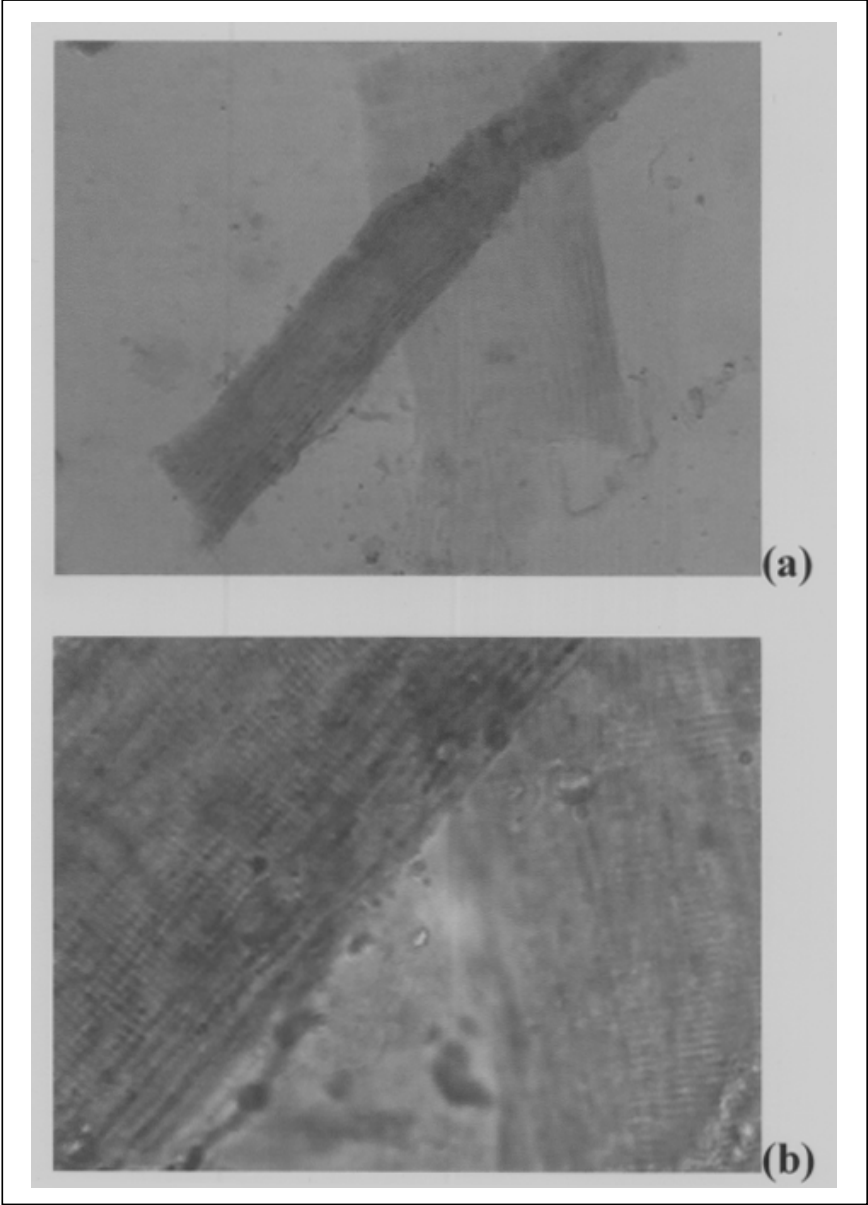

Figura 2 - Fibras musculares esqueléticas de bovinos coradas pela técnica NADH-TR (Nicotinamida Adenina Dinucleotídeo Tetrazolium Redutase). (a) fibra oxidativa fina e bem corada enquanto a fibra glicolítica, mais larga, está fracamente corada, aumento 100x; (b) fibra oxidativa corada sobre fibra glicolítica menos corada no aumento de $400 x$.

tratamento túnel de congelamento;

$\mathrm{T}=$ temperatura na porção interna do m. Longissimus dorsi.

Ciência Rural, v.36, n.6, nov-dez, 2006. 
A essa equação, correspondem os seguintes valores: $\mathrm{R}$ múltiplo $=0,890142 ; \mathrm{R}^{2}=0,792352$ e $\mathrm{R}^{2}$ ajustado $=0,746208$. O erro padrão encontrado foi de 0,165232 , em 12 observações.

EQUAÇÃO 2 (refrigeração lenta): $y_{c}=7,103-0,465 \mathrm{pH}$ $-0,138 \mathrm{~T}$, onde:

$\mathrm{y}_{\mathrm{c}}=$ comprimento de sarcômero para amostras do tratamento câmara de resfriamento;

$\mathrm{T}=$ temperatura na porção interna do $\mathrm{m}$. Longissimus dorsi.

A essa equação correspondem os seguintes valores: $\mathrm{R}$ múltiplo $=0,619398 ; \mathrm{R}^{2}=0,383654$ e $\mathrm{R}^{2}$ ajustado $=0,246688$. O erro padrão encontrado foi de 0,210046, em 12 observações.

Observando-se os valores de $\mathrm{R}^{2}$ ajustados nas duas equações e considerando-se que o $\mathrm{pH}$ variou muito pouco nos dois tratamentos, foi verificado que a equação relacionada ao túnel de congelamento foi capaz de explicar aproximadamente $75 \%$ das relações entre os valores de temperatura muscular e o comprimento de sarcômero. Enquanto isso, a equação associada à câmara de resfriamento relacionou apenas cerca de $25 \%$ dos pares de dados comprimento de sarcômero e temperatura no m. Longissimus dorsi. Isso sugere que velocidades mais lentas de resfriamento não apresentam influência relevante no grau de contração do músculo, mas um resfriamento excessivamente rápido, como ocorre rotineiramente no manejo industrial das carcaças, nos frigoríficos, pode ser o principal fator determinante do encurtamento do tecido muscular.

\section{CONCLUSÃO}

$\mathrm{O}$ método NADH-TR foi eficiente em caracterizar o encurtamento pelo frio em amostras do m. Longissimus dorsi de bovinos, podendo reduzir os erros dos métodos convencionais, que consideram ambas as fibras nas mensurações do comprimento do sarcômero, no músculo esquelético.

\section{AGRADECIMENTO}

Os autores agradecem à Fundação para o Desenvolvimento da Unesp - Fundunep, pelo apoio financeiro e ao Frigorífico Friboi Ltda., de Andradina/SP.

\section{REFERÊNCIAS}

AOAC - ASSOCIATION OF OFFICIAL ANALYTICAL CHEMISTS. Official methods of analysis. 16.ed. Maryland: Association of Official Analytical Chemists, 1997. 202p.

BRASIL. Portaria $n^{\circ} 612$, de 5 de Outubro de 1989. Sistema Nacional de Tipificação de Carcaças Bovinas. Brasília, 1989.
BRASIL. Decreto lei n. 2244, de 5 de Junho de 1997. Regulamento de Inspeção Industrial e Sanitária de Produtos de Origem Animal. Brasília, 1997.

FELÍCIO, P.E. Fatores ante e post-mortem que influenciam na qualidade da carne vermelha. In: REUNIÃO ANUAL DA SOCIEDADE BRASILEIRA DE ZOOTECNIA, 1993, Rio de Janeiro, RJ. Anais... Rio de Janeiro: Sociedade Brasileira de Zootecnia, 1993. p.2-10.

HANNULA, T.; POULANNE, E. The effect of cooling rate on beef tenderness. The significance of $\mathrm{pH}$ at $7^{\circ} \mathrm{C}$. Meat Science, v.67, n.3, p.403-408, 2004.

HEINEMANN, R.J.B.et al. Método simples para estimar encurtamento pelo frio em carne bovina. Ciência Rural, v.32, n.2, p.335-339, 2002.

HERTZMAN, C. et al. The influence of high temperature, type of muscle and electrical stimulation on the course of rigor, ageing and tenderness of beef muscles. Meat Science, v.35, n.1, p.119-141, 1993

HWANG, I. H. et al. Effects of muscle shortening and proteolysis on Warner-Bratzler shear force in beef longissimus and semitendinosus. Meat Science, v.68, n.3, p.497-505, 2004 .

LOCKER, R.H.; HAGYARD, C.J.A. A cold shortening effect in beef muscles. Journal Science Food Agricultural, v.14, n.2, p.787-793, 1963.

KOOHMARAIE, M. Muscle proteinases and meat aging. Meat Science, v.36, n.1, p 93-104, 1994.

OLSSON, U. et al. The influence of low temperature, type of muscle and electrical stimulation on the course of rigor mortis, ageing and tenderness of beef muscles. Meat Science, v.37, n.1, p.115-131, 1994.

PARRISH, F.C. et al. Effect of postmortem conditions on certain chemical, morphological and organoleptic properties of bovine muscle. Journal of Food Science, v.38, p.690$695,1973$.

POSTE, L.M. et al. Correlations of sensory and instrumental meat tenderness value as affected by sampling techniques. Food Quality and Preference, v.4, p.207-214, 1993.

RHEE, M.S. et al. Variation in palatability and biochemical traits within and among eleven beef. Journal of Animal Science, v.82, n.2, p.534-550, 2004.

SAS INSTITUTE INC. SAS User's Guide: Statistics, Rev.6. $12^{\text {th }}$. Cary: SAS Institute, 1997. 1167p.

SILVA, R.H.G. Análise eletromiográfica e morfológica dos músculos masséteres de macacos-prego (Cebus apella) submetidos à secção unilateral do nervo massetérico. 1999. 120f. T ese (Doutorado em Cirurgia Buco-Maxilo- Facial) - Faculdade de Odontologia de Araçatuba, Universidade Estadual Paulista.

WHEELER, T.L. et al. Sampling methodology for relating sarcomere length, collagen concentration, and the extent of postmortem proteolysis to beef and pork longissimus tenderness. Journal of Animal Science, v.80, n.4, p.982-987, 2002. 